\title{
Terapia Comunitária como intervenção na Saúde Mental
}

\author{
Francinete Alves de O. Giffoni ${ }^{1}$; Anny Caroline Costa Vieira ${ }^{2}$; Ana Bruna Macêdo Matos ${ }^{3}$; Jailma dos Santos Barbosa ${ }^{4}$
}

\begin{abstract}
Resumo: A saúde no Brasil tem passado por diversas mudanças, dentre elas, a aprovação de uma Política Nacional que abrange Práticas Integrativas e Complementares (PNPIC), desde 2006 e, que dá subsídios para se estender a Terapia Comunitária como prática em saúde. Esta modalidade já acontece há bem mais de vinte anos em Fortaleza e, em outras regiões do país. A Terapia Comunitária é uma forma de promoção da saúde orientada para o cuidado emocional. Historicamente tem Início na comunidade do Pirambu em Fortaleza, estado do Ceará. Trata-se de um estudo de natureza descritiva, quantitativa e qualitativa. Participaram 26 pessoas em tratamento no Centro de Atenção Psicossocial - CAPS em Barbalha, estado do Ceará. Participantes da proposta de Terapia Comunitária, implantada enquanto proposta experimental neste Centro. As entrevistas foram realizadas nos momentos dos encontros. O instrumento constou de um questionário, que foi aplicado individualmente. Os resultados demonstraram o seguinte: Quanto as concepções de Terapia Comunitária, uma categoria: Positiva, com três subcategorias: Bem estar, Encontro social e Mudança. Quanto a percepção das principais mudanças proporcionadas através das sessões de Terapia comunitária, duas categorias sobressaíram-se: Melhoria no estado mental e Melhoria nas atitudes. Finalmente, quanto a percepção dos motivos para continuar participando das sessões de Terapia comunitária, duas categorias foram eliciadas: Melhorar a saúde e relacionamentos saudáveis. Conforme verificado, ao participar das sessões de Terapia Comunitária, os entrevistados parecem perceber a importância da mudança da própria atitude no seu processo de cura. Portanto, participar da Terapia Comunitária produziu uma ruptura no cotidiano dos participantes, que relataram a ocorrência de mudanças em suas vidas durante esse período, abrangendo mudanças no estado emocional e mudanças de atitude nas relações familiares, conjugais, amorosas e sociais.
\end{abstract}

Palavras - Chave: Terapia Comunitária, Saúde, Saúde Mental.

\section{Community Therapy as Intervention in Mental Health}

\begin{abstract}
Health in Brazil has undergone several changes, among them the adoption of a National Policy that covers Integrative and Complementary Practices (PNPIC) since 2006 and that gives subsidies to extend the Community Therapy as a health practice. This mode has been going on for over twenty years in Singapore and other parts of the country. The Community Therapy is a form of health promotion oriented towards emotional care. Has historically started in the Pirambu community in Fortaleza, state of Ceará. It is a descriptive, quantitative and qualitative study. 26 people participated in treatment at the Psychosocial Care Center - CAPS in Barbalha, Ceará state. Participants of the proposed Community Therapy, implemented in the center as proposed in this experimental. Interviews were conducted during the meeting sessions. The instrument consisted of a questionnaire, which was applied individually. The results showed the following: The conceptions of Community Therapy, a category: positive, with three subcategories: Well being, social Meeting and Change. As the perception of the main changes provided through community therapy sessions, two categories stood out: Improvement in mental status and improvement in attitudes. Finally, as the perception of reasons to continue participating in community therapy sessions, two categories were elicited: Improving health and healthy relationships. As noted, to participate in the Community Therapy sessions, respondents seem to realize the importance of change of attitude in their own healing process. So joining the Community Therapy produced a break in the daily lives of the participants, who reported the occurrence of changes in their lives during this period, including changes in emotional state and attitude changes in family, marriage, loving and social relations.
\end{abstract}

Keywords: Health, Mental Health, Community Therapy.

\footnotetext{
1 Médica Psiquiatra. Mestrado em avaliação educacional. Doutorado em educação pela Universidade Federal do Ceará. francinetealves@gmail.com;

${ }^{2}$ Graduação em Fisioterapia pela Faculdade Leão Sampaio. E-mail: anny_carol_15@hotmail.com;

${ }^{3}$ Graduada em Enfermagem pela Faculdade Leão Sampaio e Especialista em Urgência e Emergência pela Faculdades Integradas de Cruzeiro - SP. anabrunamacedo@hotmail.com;

${ }^{4}$ Graduada em Enfermagem. e Pedagogia. Especialista em Gestão em Saúde, Especialista em Auditoria em Saúde, Especialista em Enfermagem do Trabalho, Especialista em Saúde da Família, Especialista em Gestão Escolar. E-mail: jailmaestre@ hotmail.com;
} 


\section{Introdução}

A saúde no Brasil tem passado por diversas mudanças, dentre elas, a aprovação de uma Política Nacional que abrange Práticas Integrativas e Complementares (PNPIC), desde 2006 e, que dá subsídios para se estender a Terapia Comunitária como prática em saúde. Esta modalidade já acontece há bem mais de vinte anos em Fortaleza e, em outras regiões do país.

Em 2008, foi firmado um convênio entre a Fundação Cearense de Pesquisa e Cultura e o Ministério da Saúde (MS), para a implementação da Terapia Comunitária na Assistência Básica à Saúde através do Sistema Único de Saúde (SUS). Tal projeto teve o objetivo de desenvolver, tanto nos profissionais de saúde como nas lideranças comunitárias, algumas das competências necessárias para que pudessem lidar com o sofrimento de algumas demandas psicossociais, bem como de promover redes de apoio, de forma a ampliar a capacidade de resolutividade da Atenção Básica. Agregar as atividades da Terapia Comunitária no SUS, atende às demandas que estão expressas na Política Nacional de Atenção Básica, dentre elas: a)implementar diretrizes da Política Nacional de Humanização - PNH, via acolhimento; b)realizar uma escuta mais qualificada sobre as necessidades dos usuários; c)estabelecimento de vínculos entre usuários e serviços de assistência (MINISTÉRIO DA SAÚDE, 2008). Adicionalmente, responde aos interesses da Política Nacional de Promoção da Saúde, que visa o fortalecimento da participação social, entendendo este como fundamental para consecução de resultados em termos de promoção da saúde, principalmente o empoderamento individual, privilegiando práticas de saúde já adotadas pelas comunidades, ajustadas à realidade do Brasil (MINISTÉRIO DA SAÚDE, 2008).

A Terapia Comunitária é uma forma de promoção da saúde criada pelo Professor Adalberto Barreto, professor da Universidade Federal do Ceará, orientada para o cuidado emocional. Historicamente tem Início na comunidade do Pirambu em Fortaleza, estado do Ceará, posteriormente tornando-se bastante conhecida por conta dos resultados satisfatórios apresentados. Surgiu como mais instrumento para se trabalhar a saúde, levando-se em conta as questões sociais e emocionais envolvidas no processo saúde-doença, com a ajuda das pessoas na comunidade onde esta se insere (FERREIRA FILHO e DIAS, 2006).

Trata-se de uma forma de trabalho que agrega a escuta empática e o acolhimento na promoção da saúde mental. Deve-se também ressaltar, o baixo custo envolvido associados aos resultados positivos, em termos de uma maior compreensão do quadro individual. Leva em conta as dificuldades e preocupações sobre a saúde numa determinada comunidade. Estas são em muito superiores às mais tradicionais formas de terapia.

As práticas mais comuns de saúde, tem evoluído baseando-se na localização física e numa classificação das doenças que busca suas evidências no corpo físico. Esta forma tem sido muito 
debatida, enquanto maneira de entendimento da saúde geral, isto é, uma visão da saúde como ausência de doença (ENUMO, 2003).

No entendimento de Caponi (1997), o conceito de saúde, vai além dos limites da compreensão do que apregoa a medicina tradicional. Que a saúde é experiência pessoal e subjetiva, que nos habilita saber o que se passa em nosso próprio corpo.

O conceito de saúde que é proposto pela Organização Mundial de Saúde (OMS), a entende como um completo estado de bem-estar, social, físico e psicológico. E mesmo este, tem sido amplamente discutido, justo por considerar a saúde como um estado, não como um processo (DEJOURS, 1986; CAPONI, 1997; SARRIERA, MOREIRA, ROCHA, BONATO, DUSO \& PRIKLADNICKI, 2003). Outros autores defendem o caráter de complexidade da saúde como um fenômeno. Tais mudanças determinaram em uma nova concepção da saúde. Esta nova concepção, considera outros determinantes, tais como: o meio ambiente em que se vive, a alimentação adotada, o acesso aos bens de consumo, saneamento básico, questões relacionadas ao trabalho, justiça social, renda e educação (CARVALHO, 1996; BUSS, 2000).

Quando se busca as Representações de tais fenômenos, o binômio saúde-doença passa a ser entendido de forma mais ampla, incluindo as relações sociais estabelecidas, que são definidoras de múltiplas causas de doenças. São fatores contributivos: processo de produção, religiosidade, acesso a saúde, valores éticos e morais, dentre outros (TRINDADE, 1996).

Outros autores como Enumo (2003), enfatizam a possibilidade do modo de viver do doente, como uma forma de desmistificar o discurso médico. Dessa maneira, as Representações da pessoa, teria maior significado para este estudo, enquanto um revelador das percepções do enfermo, sobre seu próprio estado ou doença. Neste caso, segundo Trindade (1996), a concepção do que vem a ser doença, sai do lugar comum do saber médico, para restaurar a importância do pensar individual e autônomo do paciente, levando em conta sua lógica e sua coerência próprias, priorizando seu discurso para a eficácia do tratamento.

O conhecimento das representações individuais das pessoas, torna-se útil para o planejamento de ações em saúde, pois revela crenças e conhecimentos que podem ser acrescidos ao saber médico, para que possa ser mais eficaz nas suas investidas preventivas.

\section{Método}

Estudo de natureza descritiva, quantitativa e qualitativa. Participaram 26 pessoas em tratamento no Centro de Atenção Psicossocial - CAPS em Barbalha, estado do Ceará. Participantes da proposta de Terapia Comunitária, implantada enquanto proposta experimental neste Centro. 
Os participantes foram autorizados pelos médicos e profissionais, ora em acompanhamento dos mesmos. Estes informaram que os mesmos estariam em condições satisfatórias para responderem os questionários, uma vez que se mostravam em condições clínicas mais favoráveis na época da coleta dos dados.

As entrevistas foram realizadas nos momentos dos encontros da Terapia Comunitária, por facilitar as entrevistas e o acesso às famílias. O contato inicial com os profissionais na instituição, foi necessário para autorização do contato junto aos respondentes e suas famílias.

\section{Instrumento de coleta de dados}

O instrumento constou de um questionário, que foi aplicado individualmente, envolvendo questões como tempo de tratamento com a Terapia comunitária, a percepção da evolução do tratamento, percepção das mudanças na vida e na convivência familiar, expectativas de vida e quanto a cura, bem como as representações sociais de Terapia comunitária.

As questões que foram abordadas levaram em conta, tanto a prática de atendimento no Centro, como os trabalhos que descrevem as crenças, os medos e as representações sociais de saúde (SCHULZE, 1995; NEME \& KATO, 2003; DÓRO e cols., 2004).

Conforme Gaskell (2003), as entrevistas semi-estruturadas são amplamente empregadas em pesquisas qualitativas, pois são capazes de fornecer dados mais básicos, para uma compreensão das complexas relações entre os indivíduos e sua situação em si. O seu objetivo é uma compreensão, o mais detalhada possível, dos valores, crenças e atitudes, bem como das motivações, quanto aos comportamentos, quando em contextos sociais mais específicos.

\section{Procedimentos de Coleta de dados}

A participação dos entrevistados aconteceu mediante consentimento livre e esclarecido, sendo adotado um termo de consentimento contendo uma descrição clara dos procedimentos de coleta dos dados e sigilo, de acordo com a resolução no 466/12, do Conselho Nacional de Saúde.

Além disso, foi também considerado a possibilidade do impacto emocional causado pela entrevista aos participantes (choro, tristeza, etc.) que, por ventura, pudessem vir a comprometer o estado geral dos respondentes. Nestes casos, a pessoa seria encaminhada ao Serviço de Psicologia na instituição, o que não chegou a acontecer. 


\section{Procedimentos de Análise dos dados}

Os dados de cada uma das entrevistas foram organizados em "unidades de significado" (os aspectos das falas dos participantes, que foram considerados como importantes, por manterem estreita relação com os objetivos da pesquisa) extraídas da análise das entrevistas (TRINDADE, 1991), aqui relacionadas: 1) Representação de Terapia Comunitária; 2) Percepção do que mudou após o tratamento; 3) Motivos que os impulsiona ainda a participar da Terapia Comunitária.

Essa forma de discussão de resultados, contribuiu para uma melhor compreensão das percepções dos respondentes, e possibilitou a identificação de eventuais variações nos elementos eliciados nas representações sociais de Terapia Comunitária.

\section{Resultados e Discussão}

\section{Concepções de Terapia Comunitária pelos sujeitos}

Uma categoria se sobressaiu: Positiva., uma vez que, em resposta a pergunta: o que representa para você a terapia Comunitária?, as respostas eliciadas fizeram referências a sentimentos e palavras positivas. Estes foram agrupados em três subcategorias, conforme a tabela 1 a seguir.

Tabela 1 - Descrição das subcategorias referentes a categoria Positivo. Referentes às Representações de Terapia Comunitária. Barbalha, 2015.

\begin{tabular}{|c|c|}
\hline $\begin{array}{l}\text { BEM-ESTAR - Neste caso, a } \\
\text { percebida como potencializadora } \\
\text { de conforto físico e emocional, } \\
\text { sentimentos de paz, amor, fe } \\
\text { quais deriva um sentimento func } \\
\text { estar. }\end{array}$ & 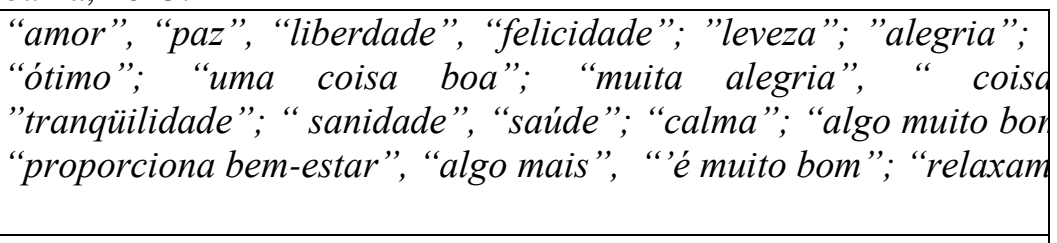 \\
\hline $\begin{array}{l}\text { ENCONTRO SOCIAL - A } \\
\text { experienciada como uma fer } \\
\text { permite ampliar as relações, } \\
\text { clima de maior intimidade e r } \\
\text { os participantes. }\end{array}$ & $\begin{array}{l}\text { "local de diálogo"; "pessoas estão a escutar"; "lugar de } \\
\text { problemas", "existem pessoas para ouvir"; "se distrai muito"; " } n \\
\text { confraternização"; "espero durante toda semana"; "distraio min } \\
\text { "não fico pensando besteiras em casa"; "ambiente de enco } \\
\text { amigos"; "Local de desabafar os problemas"; "local de conselh } \\
\text { ação de ajuda"; "um apoio para problemas". }\end{array}$ \\
\hline $\begin{array}{l}\text { MUDANÇA - Neste caso, } \\
\text { parece ter sido promotora d } \\
\text { consciência a } \quad \text { respeito } \\
\text { autoconfiança, } \\
\text { possibilidade de solução das }\end{array}$ & $\begin{array}{l}\text { "solução de dificuldade"; "desenvolvimento"; "nos ajuda"; " } \\
\text { besteiras"; "um caminho bom mental"; "para melhoria do } \\
\text { "vitória"; "atitude triste é amenizada"; }\end{array}$ \\
\hline
\end{tabular}

Fonte: Dados da Pesquisa 
Através dos discursos foi possível perceber uma conotação positiva atribuída pelos participantes aos encontros. A Terapia Comunitária pareceu-nos uma estratégia poderosa, mesmo que na duração de poucos encontros, que proporciona uma prática democrática de vivência humanizada. Neste lugar, todos parecem sentir-se como semelhantes em suas vivências.

Desta forma, é fundamental a adoção de práticas que possam auxiliar na promoção da socialização das pessoas, aumentando-lhes o bem-estar físico e psicológico.

\section{Percepção das principais mudanças proporcionadas através das sessões de Terapia comunitária}

Tabela 2 - Descrição das categorias referentes as mudanças proporcionadas através das sessões de Terapia Comunitária. Barbalha, 2015.

\begin{tabular}{|l|l|}
\hline MELHORIA NO "mais distraido", "mais feliz"; "mais segurança psicológica; "mg \\
MENTAL & $\begin{array}{l}\text { "alivio nos problemas"; "procuro um caminho melhor"; " } \\
\text { recarregar as baterias"; "tornei-me mais leve"; "melhorei os pro } \\
\text { nervos"; "deixei o pensamento vazio"; "ocupar a mente". }\end{array}$ \\
\hline MELHORIA NAS ATITU "mais obediente", cuidar dos pais"; "melhorar o relacionamen \\
$\begin{array}{l}\text { outras pessoas"; "mais confiança nos outros"; "não desconfiar d } \\
\text { todos"; "conviver melhor fora da residencia"; "maneira corr } \\
\text { relacionar com as outras pessoas", "fazer o que é certo". }\end{array}$
\end{tabular}

Fonte: Dados da Pesquisa

\section{Percepção dos motivos para continuar participando das sessões de Terapia comunitária}

Tabela 3 - Descrição das categorias referentes aos motivos para continuar participando das sessões de Terapia Comunitária. Barbalha, 2015.

\begin{tabular}{|l|l|}
\hline MELHORAR A SAÚDE & $\begin{array}{l}\text { "participar de atividades paralelas: como artes, passeios"; "dese } \\
\text { boa"; "ajudar o outro", "salvar a vida"; "necessidade de tr } \\
\text { "sentir-me mais a vontade"; "ver possibilidade da cura"; "bus } \\
\text { para melhorar a saúde". }\end{array}$ \\
\hline $\begin{array}{l}\text { RELACIONAMENTOS } \\
\text { SAUDÁVEIS }\end{array}$ & $\begin{array}{l}\text { "ajudar dos membros do CAPS"; "visitar amigos para distrair"; " } \\
\text { coisas ruins"; "ouvir as pessoas"; "buscar novos amigos", "conh } \\
\text { pessoas para conversar"; "atenção em ouvir problemas do } \\
\text { "interesse no superar os problemas", "tentar ajudar". }\end{array}$ \\
\hline
\end{tabular}

Fonte: Dados da Pesquisa

Considerando as representações sociais enquanto processos, através dos quais, os objetos sociais mais próximos são significados como interdependentes, as representações sociais de Terapia Comunitária, não poderia estar dissociada de melhoria da saúde e de relacionamentos saudáveis. 
Ainda que todos os respondentes tenham buscado um tratamento médico como um meio de cura, para alguns, tal cura passou a depender também da própria eficácia da Terapia Comunitária. Isso principalmente para aqueles que demonstraram um maior vínculo com o novo tratamento.

Segundo Minayo (1994), as situações de extremo sofrimento, de desespero quando frente à doença e outros problemas, são elementos que nos motivam para a crença na cura, através de meios não convencionais.

É comum nos encontros de Terapia Comunitária as pessoas relatarem momentos de superação. E, o que aprenderam nos momentos de discussão de problemas semelhantes, quanto ao do tema escolhido. As experiências de vida, tendem a caracterizar o poder resiliente de cada um, fazendo reconhecer a contribuição da TC para a manutenção da saúde mental.

Por resiliência, entenda-se um processo que excede o simples superar. Permite ao indivíduo, sair fortalecido de situações adversas, contribuindo em muito para sua saúde mental. Ela diminui a intensidade do estresse e, de sinais emocionais negativos, como depressão, ansiedade e a raiva. E, sob condições e valores próprios, lhes permite metabolizar o evento negativo e construir a partir dele.

Um dos fatores da resiliência é o respeito pelos outros e por si mesmo, fazendo com que ela seja positiva quando alcança as expectativas sociais. Assim a capacidade resiliente permite tolerar, manipular e aliviar as consequiências psicológicas, sendo efetiva não apenas para adversidades, mas para promoção da saúde mental e emocional; contribuindo dessa forma para qualidade de vida (SARRIERA et al., 2003).

Tendo em vista que a vivencia pessoal e o compartilhamento de sofrimentos são fontes de saber, as reflexões dos encontros de TC são necessárias porque elas funcionam como mola propulsora para o processo de empoderamento e resiliência.

Conforme verificado, ao participar das sessões de Terapia Comunitária, alguns entrevistados começam a perceber a importância da mudança da própria atitude no seu processo de cura, através do reconhecimento da própria impotência perante a dor, o sofrimento e/ou a doença dos outros participantes, com os quais passa a se identificar. Acreditamos que o apoio e conforto emocional oferecidos pela Terapia Comunitária pode ser uma das explicações para o fortalecimento do vínculo afetivo com o grupo, proporcionadores de uma mudança de estado mental, como relatado por alguns participantes.

Verificamos que participar da Terapia Comunitária produziu uma ruptura no cotidiano dos participantes, que relataram a ocorrência de mudanças em suas vidas durante esse período, abrangendo mudanças no estado emocional e mudanças de atitude nas relações familiares, conjugais, amorosas e sociais. Com o decorrer do tratamento, a busca por maiores informações sobre os problemas comuns, junto ao grupo e no relacionamento individual com outras pessoas com as mesmas dificuldades, parece ter elucidado novos parâmetros de reflexão para os entrevistados, os quais, a partir disso, 
passaram a adotar novas atitudes em sua vida de relacionamentos e para consigo mesmos. Essa nova forma de viver parece ter adquirido um valor positivo para essas pessoas.

A maioria dos entrevistados atribuiu a mesma valoração a algumas das mudanças relatadas como, por exemplo, a valorização da família e do convívio social, evidenciada nas seguintes falas: "mais obediente aos pais"; "melhorou o relacionamento com outras pessoas"; "comecei a adquirir confiança nos outros"; "não desconfiar de todos"; "consegui conviver fora da residência"; "maneira correta de se relacionar com as outras pessoas".

Isso não quer dizer que essas mudanças foram experienciadas de forma idêntica, mas o significado compartilhado não só nos mostra como essas mudanças são sentidas e avaliadas, como pode indicar a existência de elementos comuns, característicos do processo de estar em tratamento.

Percebe-se, assim, que outros elementos foram incorporados à representação social de Terapia Comunitária a partir do momento em que, o motivo para participar das sessões passa a ter uma função importante no tratamento: Melhorar a saúde e Ressignificação. A busca pela saúde parece ter promovido um retorno a uma condição de vida mais consciente. Enfrentando mais ativamente as suas dificuldades no tratamento, a saúde passa a ter um novo significado, como sentir que estar vivo, presente, fazendo parte de uma rede social, como relatado nas seguintes falas dos participantes: "realização de atividades paralelas: pintura, passeios"; "possibilidade de salvar a vida"; "conscientização da necessidade de tratamento"; "sentir-se mais a vontade"; "rever os membros do CAPS"; "sair de casa para distrair"; "deixar de pensar coisas ruins"; "busca de pessoas para conversar"; "interesse em superar os problemas".

\section{Conclusão}

Nesse estudo foi verificado que o problema mais freqüente nos idoso é o estresse, que a estratégia de enfrentamento mais utilizada é o fortalecimento da espiritualidade, e que através dos discursos dos idosos registrados nas fichas é possível perceber os benefícios alcançados e as mudanças que ocorreram em suas vidas após a participação nos encontros de TC. Encontrou-se, além disso, o perfil dos idosos participantes da TC, como sendo a idade maior de 60 anos, a maioria do sexo feminino e moradoras de comunidades carentes.

Os temas encontrados caracterizam a realidade local e acarretam insegurança e instabilidade sócio-afetiva numa fase da vida que requer atenção e cuidados redobrados; além disso, a correlação entre eles nos remete a idéia de uma cascata de problemas que podem surgir após o aparecimento de alguma dificuldade, potencializando o comprometimento mental. As estratégias de enfretamento desvendadas caracterizam o poder resiliente em cada participante e demonstram a importância do saber popular, fazendo valer a cultura local que tem por essência o senso comum. 
Esses achados permitiram conhecer a importância da Terapia Comunitária como estratégia de cuidado, além de fomentar subsídios para ações mais humanizadas, tendo em mente que, é respeitando os aspectos culturais de cada indivíduo e levando em consideração seus conhecimentos e experiências prévias, em termos de suas próprias condições de prevenir a saúde mental

Foi possível evidenciar a importância da Terapia Comunitária, no contexto do tratamento, quando a mesma evocou nos participantes uma representação positiva, relacionada ao Bem-estar, a Encontro social e Mudança possibilitando um novo modo de pensar suas atitudes e práticas sociais.

As variações encontradas nos elementos das representações sociais de Terapia Comunitária vêm mostrar a possibilidade da saúde representada de forma múltipla, não restrita à ausência de doença, incorporando outros determinantes, como socialização e ressignificação, que são valores sociais importantes para que o indivíduo se reconheça como sujeito.

Esperamos que essas considerações possam orientar intervenções no campo da saúde mental, não apenas dos profissionais de medicina ou psicologia, mas também de outras áreas do conhecimento que têm como objetivo a promoção da saúde, para que se possa valorizá-la também como um direito a que todos devem ter acesso.

\section{Referências}

BUSS, P. M. Promoção da saúde e qualidade de vida. Ciência \& Saúde Coletiva, 2000. 5 (1), 163-177.

CANGUILHEM, G. O normal e o patológico. Rio de Janeiro: Forense Universitária, 1990.

CAPONI, S. Georges Canguilhem y el estatuto epistemológico del concepto de salud. História, Ciência, Saúde, 1997. 4 (2), 287-387.

CARVALHO, A. I. Da saúde pública às políticas saudáveis: Saúde e cidadania na pós-modernidade. Ciência \& Saúde Coletiva, 1996. 1 (1), 104-121.

DEJOURS, C. Por um novo conceito de saúde. Revista Brasileira de Saúde Ocupacional, 1986. 14 (54), 07-11.

DÓRO, M. P.; PASQUINI, R.; MEDEIROS, C. R.; BITENCOURT, M. A. \& MOURA, G. L. O câncer e sua representação simbólica. Psicologia: ciência e profissão, 2004. 24, (2), 120-133.

ENUMO, S. R. F. Pesquisas sobre Psicologia \& Saúde: uma proposta de análise. Em A. N. Andrade \& Z. A. Trindade (Orgs.). Psicologia e Saúde: Um campo em construção. 2003. (pp. 11-31). São Paulo: Casa do Psicólogo.

FERREIRA FILHO, MO; DIAS, MD. Terapia Comunitária: uma ação básica de saúde mental. João Pessoa: Projeto de Extensão (PROBEX)/UFPB; 2006.

GASKELL, G. Entrevistas individuais e grupais. In M. W. Bauer \& G. Gaskell (Orgs.). Pesquisa qualitativa com texto, som e imagem. 2003. (pp. 64-89). Petrópolis: Vozes. 
MINAYO, M. C. S. Representação da cura no catolicismo popular. Em P. C. Alves \& M. C. S. Minayo (Orgs.). Saúde e doença: um olhar antropológico (pp.57-71). Rio de Janeiro: Editora Fiocruz, 1994.

MINISTÉRIO DA SAÚDE - Fundação Oswaldo Cruz. Diretrizes e normas regulamentadoras de pesquisas envolvendo seres humanos: Resolução 196/96 do Conselho Nacional de Saúde. Rio de Janeiro: Fundação Oswaldo Cruz, 1998.

NEME, C. M. B. \& KATO, S. Mulheres com câncer de mama: Crenças sobre a doença e temores quanto ao tratamento. Em C. M. B. Nome \& O. M. P. R. Rodrigues (Orgs.). Psicologia da saúde: Perspectivas interdisciplinares. 2003. (pp. 125-148). São Carlos: Rima.

SARRIERA, J. C.; MOREIRA, M. C.; ROCHA, K. B.; BONATO, T. N.; DUSO, R. \& PRIKLADNICKI, S. (2003). Paradigmas em psicologia: Compreensões acerca da saúde e dos estudos epidemiológicos. Psicologia e Sociedade. 2003. 15 (2), 88-100.

SCHULZE, C. M. N. As representações sociais de pacientes portadores de câncer. Em M.J.P. Spink (Org.). O conhecimento no cotidiano: As representações sociais na perspectiva da psicologia social (pp. 266-279). São Paulo: Brasiliense, 1995.

TRINDADE, Z. A. Representações sociais da paternidade e da maternidade: Implicações no processo de aconselhamento genético. Tese de Doutorado. Instituto de Psicologia/USP, São Paulo/SP, 1991.

TRINDADE, Z. A. Representação social: "modo de conhecer" no cenário da saúde. Em C. Camino; Z. A. Trindade (Orgs.). Cognição social e juizo moral (pp. 45-59). Rio de Janeiro: Associação Nacional de Pesquisa e Pós-Graduação em Psicologia - ANPEPP, 1996.

\section{Como citar este artigo (Formato ABNT):}

GIFFONI, F.A.O.; VIEIRA, A.C.C.; MATOS, A.B.M.; BARBOSA, J.S. Terapia Comunitária como intervenção na Saúde Mental. Id on Line Revista de Psicologia, Novembro de 2015, vol.9, n.28. p. 61-70. ISSN 19811189.

Recebido: $15 / 08 / 2015$

Aceito: 1610/2015 